\title{
Massive Traumatic Hematoma Localized in the Basal Ganglia
}

\author{
Minotu Shigemori, Takashi Tokutomi, ${ }^{*}$ Morihisa Shirahama, \\ Kunitada HARA and Fumihito Yamamoto \\ Division of Neurosurgery, Omuta City Hospital, Omuta 836 \\ * Department of Neurosurgery, Kurume University \\ School of Medicine
}

\begin{abstract}
Summary
A case of massive traumatic hematoma of the basal ganglia which simulated a spontaneous intracerebral hematoma and was successfully treated is reported. The possible mechanism of production of these lesions following head injury is also discussed.
\end{abstract}

Key words: Head injury, basal ganglia, intracerebral hematoma, shearing injury

\section{Introduction}

Although traumatic hematomas in the region of the basal ganglia have been found to be fairly uncommon among traumatic intracerebral hematomas, ${ }^{2,8)}$ recent reports ${ }^{1,5,7)}$ have suggested that such hematomas are more common than previously thought with the recent advent of computerized tomography (CT). There is, however, still a controversy as to the pathological mechanism producing these lesions. ${ }^{1,3,9,10)}$ Such hematomas often result in a medicolegal problem concerning whether they are spontaneous or traumatic in origin in patients beyond middle age. ${ }^{2,3,5}$ ) A case with a massive hematoma of the basal ganglia produced by severe head injury which was successfully treated and its possible mechanism are described.

\section{Case Report}

A 16-year-old school boy who had no demonstrable predisposing abnormalities was admitted on November 30, 1979, approximately 30 minutes after being involved in an automobile accident. He was thrown from his motorcycle and struck his head in the occipital region. He was semicomatose, with a right dilated pupil and a decerebrate posture mainly on the left side. The pupils did not react well to light. His blood pressure was $140 / 90 \mathrm{mmHg}$, pulse rate
$80 /$ minute and respiratory rate $25 /$ minute. There was a left facial and right occipital contusions. X-ray film of the skull showed a right occipital linear fracture and diastasis of the right lambdoid suture. An emergency CT scan demonstrated a large massive hematoma localized in the region of the basal ganglia involving the lenticular nucleus and the external capsule. The medial edge of the hemorrhagic lesion corresponding to the pallidum was irregular and isolated from the antero-lateral discrete mass. A small hemorrhagic focus at the site of the splenium and a subarachnoid hemorrhage in the left sylvian fissure were also noted (Figs. 1 and 2). Right carotid angiography was then performed to rule out the presence of any cerebrovascular anomalies. This indicated a round shift of the anterior cerebral artery and lateral displacement of the insular portion of the middle cerebral artery showing a centrally located avascular mass. The lenticulostriate arteries were all faint and poorly opacified. The cisternal portion of the anterior choroidal artery was stretched upward without the initial downward curve. There were no demonstrable vascular anomalies such as a cerebral aneurysm or arteriovenous malformation (Fig. 3A and B).

An emergency operation for evacuation of the hematoma was then performed. On opening of the dura mater following right frontotemporal craniotomy, a tense, otherwise normal looking brain was found. A puncture through 


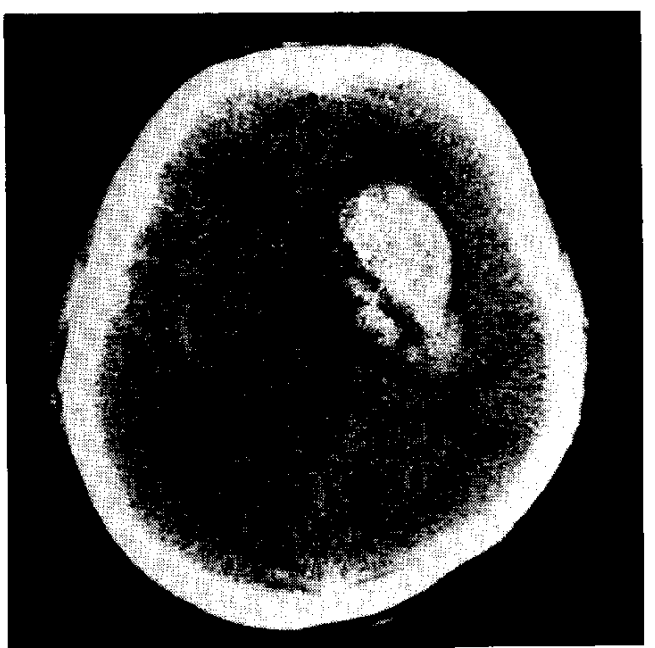

Fig. 1 CT scan on admission shows a massive hematoma localized in the right basal ganglia and a mild subarachnoid hemorrhage at the left sylvian fissure. The medial portion of the lesion shows an isolated, irregular area of blood density suggestive of a contusion of the pallidum.

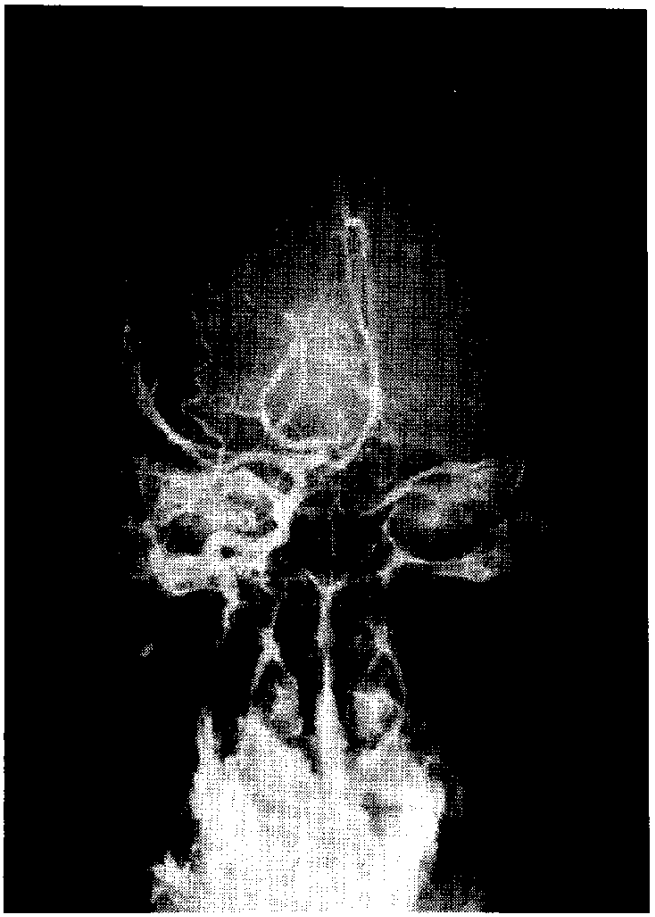

A the frontal convolution yielded dark bloody fluid at a depth of $3 \mathrm{~cm}$. Most of the large deep-seated hematoma localized entirely medial

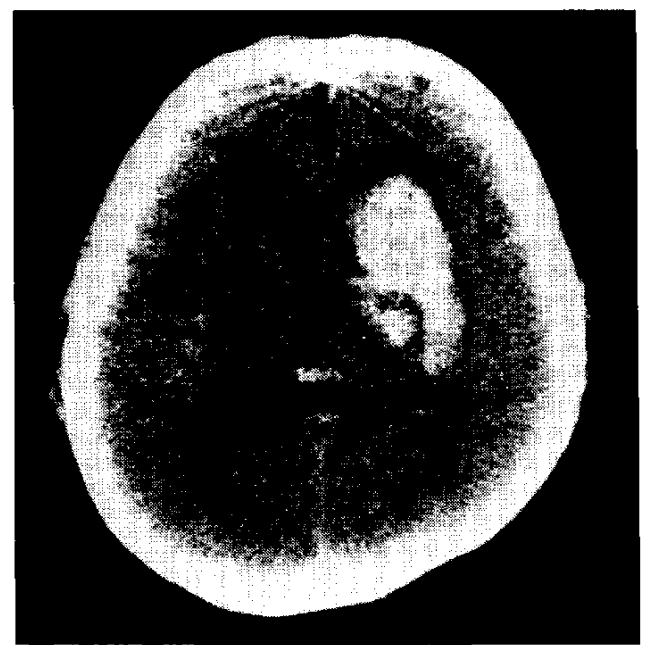

Fig. 2 A higher cut of the CT scan reveals a small hemorrhagic focus at the site of the splenium in addition to a massive hematoma of the basal ganglia.

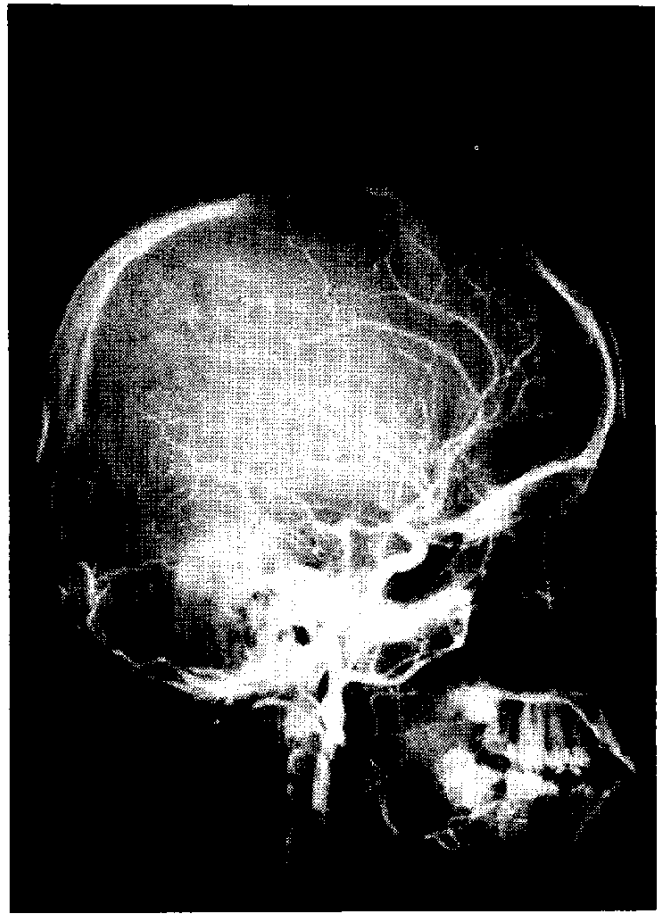

B

Fig. 3 A and B: Right carotid angiogram on admission shows a centrally located avascular mass. Poor opacification of all lenticulostriate arteries on the right side and stretching of the cisternal portion of the anterior choroidal artery are also demonstrated. There is no demonstrable vascular abnormality. 
to the insula was evacuated by suction through a frontal cortical incision. The frontotemporal bone flap was removed following hemostasis for fear of secondary brain edema. The evacuated hematoma clots weighed approximately $70 \mathrm{~g}$. Postoperatively, he remained stuporous, but gradually improved and the extensor rigidity of the limbs also became mild. Although involuntary movement simulating choreoathetosis on the right upper limb developed transiently, his consciousness became alert and he recovered without mental deficits except for left spastic hemiparesis 3 months after the operation.

\section{Discussion}

Traumatic intracerebral hemorrhages have been described as being essentially a coupcontrecoup effect, with the injury being sustained when the head is in motion. ${ }^{3)}$ As the cause of traumatic lesions in the cerebral white matter or the juncture between the cortex and white matter following severe head injury, Holbourn $^{6)}$ stressed the role of shearing injury. $\mathrm{He}$ indicated that strains resulted at the juncture between the white and gray matter when the skull was rotated by an external force. Courville and Blomquist ${ }^{3)}$ reported seven cases of traumatic hemorrhages in the ganglionic region and noted that these were located in the zone of the lenticular nucleus and external capsule, and the bleeding was slight and usually multiple. Therefore, they believed that massive hematomas acting as a space occupying lesion were always spontaneous in origin. However it was clearly demonstrated by the works of Mosberg ${ }^{10)}$ and Lindenberg, 9) that massive hematomas were produced by severe head injury and the tentorial edge played a significant role in these lesions. They also gave several prerequisites for such lesions. The conditions should be such that the impact is received by the moving and often accelerated head with a magnitude sufficient to deform the skull, the impact area must be the forehead, convexity or occiput, and the impact force must be directed towards the tentorium. Under these circumstances, the central portions of the brain are contused with a shifting forward into the tentorial opening. This leads to shearing within the tissues resulting in the contusion hemorrhage in the central structure of the brain. They further showed the significance of an injury to one of the pallidum branches of the anterior choroidal artery and possible involvement of the striate-body branches of the middle cerebral artery as the cause of large hematomas in this location.

The evidence in this young patient might be sufficient to prove the traumatic origin according to their descriptions. It can be assumed that the tearing of small arteries in the central structures of the brain caused by shearing forces among the different tissues might bring about a massive hematoma involving both the putamen and pallidum. From the location of the main hematoma demonstrated on the CT scan in this case, the lenticulostriate arteries seem to be the major bleeding vessels although it is unknown whether or not the branches of the anterior choroidal artery were involved concomitantly. Angiographic demonstration of poor filling of the right lenticulostriate arteries suggest these events. A contusion hemorrhage at the pallidum might also be indicated because of its "fluffy" edge around the lesion which was reported as characteristic of traumatic origin. ${ }^{4)}$ An eccentric hemorrhagic lesion at the site of the splenium may also suggest the role of shearing forces because hemorrhagic lesions in the corpus callosum, around the third ventricular region or midbrain, were described as the manifestations of shearing injuries of the cerebral white matter. ${ }^{11)}$ In addition, these paraventricular structures are also known to be displaced through the tentorial notch, and a contusion or small hemorrhages in these locations should be associated with a massive hematoma of the basal ganglia. ${ }^{9,10)}$

Since these patients demonstrate severe neurological deficits such as immediate onset of coma, decerebration or hemiparesis as seen in the present case ${ }^{3,11)}$ and their sequelae, an emergency operation in addition to intensive treatment is mandatory through a large craniotomy when the hematoma is sufficiently large to act as a space occupying lesion simulating an spontaneous intracerebral hematoma on CT scans. 


\section{Acknowledgment}

We wish to thank to Prof. Shinken Kuramoto, Department of Neurosurgery, Kurume University, for his review of the manuscript.

\section{References}

1) Akimoto, H., Maki, Y., Tajima, K., Shirai, S. and Tosa, J.: Injuries of basal ganglia following head trauma in children. Nervous System in Children 4: 215-224, 1979 (in Japanese).

2) Borovich, B., Gellei, B. and Peyser, E.: Massive traumatic hematoma of the basal ganglia. Surg Neurol 3: 25-26, 1975.

3) Courville, C. B. and Blomquist, O. A.: Traumatic intracerebral hemorrhage with particular reference to its pathogenesis and its relation to "delayed traumatic apoplexy". Arch Surg 41: 1-28, 1940.

4) French, B. N. and Dublin, A. B.: The value of computerized tomography in the management of 1,000 consecutive head injuries. Surg Neurol 7: 171-183, 1977.

5) Hara, M., Shiogai, T., Tamagawa, T.,
Yokota, H., Okada, J. and Akeuchi, K.: Three cases of traumatic intracerebral hematoma with ventricular hemorrhage. Neurol Surg (Tokyo) 8: 295-299, 1980 (in Japanese).

6) Holbourn, A. H. S.: The mechanics of brain injuries. Br Med Bull 3: 147-149, 1954.

7) Hondo, H., Yoshijima, S., Kusaka, K. and Matsumoto, K.: Two cases of traumatic intracerebral hemorrhage in the basal ganglia. Proceedings of the 1st Conference of the Japanese Society of Neurotraumatology 1: 101-105, 1978 (in Japanese).

8) Jamieson, K. G. and Yellard, J. D. N.: Traumatic intracerebral hematoma: Report of 63 surgically treated cases. $J$ Neurosurg 37: 528-532, 1972.

9) Lindenberg, R.: Significance of the tentorium in head injuries from blunt forces. Clin Neurosurg 12: 129-142, 1966.

10) Mosberg, W. H. and Lindenberg, R.: Traumatic hemorrhage from the anterior choroidal artery. J Neurosurg 16: 209-221, 1959.

11) Zimmerman, R. A., Bilaniuk, L. T. and Genneralli, T.: Computed tomography of shearing injuries of the cerebral white matter. Radiology 127: 393-396, 1978. 